\title{
Mitteilungen der DGG
}

Gefässchirurgie 2020 $25: 305-307$

https://doi.org/10.1007/s00772-020-00654-z

(c) Springer Medizin Verlag GmbH, ein Teil von Springer Nature 2020

Korrespondenzanschriften für die Mitteilungen der DGG

Deutsche Gesellschaft für Gefäßchirurgie und Gefäßmedizin Geschäftsstelle

Robert-Koch-Platz 9, 10115 Berlin

T.: 030/28099099-0

F.: 030/28099099-9

sekretariat@gefaesschirurgie.de

Sekretär: PD Dr. F. Adili (V.i.S. d. P.)

Klinik für Gefäßmedizin - Gefäß- und Endovascularchirurgie

Grafenstraße 9

64283 Darmstadt

T.: 06151 - 1078000

F.: 06151 - 1074429

sekretaer@gefaesschirurgie.de

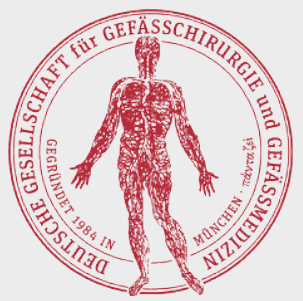

Die Inhalte der Mitteilungsseiten der Zeitschrift Gefässchirurgie liegen in der Verantwortung der entsprechenden Fachgesellschaften. Herausgeber und Verlag übernehmen keine Verantwortung für diese Rubrik.

\section{Neues aus der Geschäftsstelle}

Nach über 10 Jahren als Leiterin der Geschäftsstelle und Geschäftsführerin der Privaten Akademie beendete Frau Dr. Ing. Monique Jacobs auf eigenen Wunsch zum 30. April 2020 ihre Tätigkeit für die DGG. Während dieser Zeit hatte sie großen Anteil an der Weiterentwicklung und Professionalisierung unserer

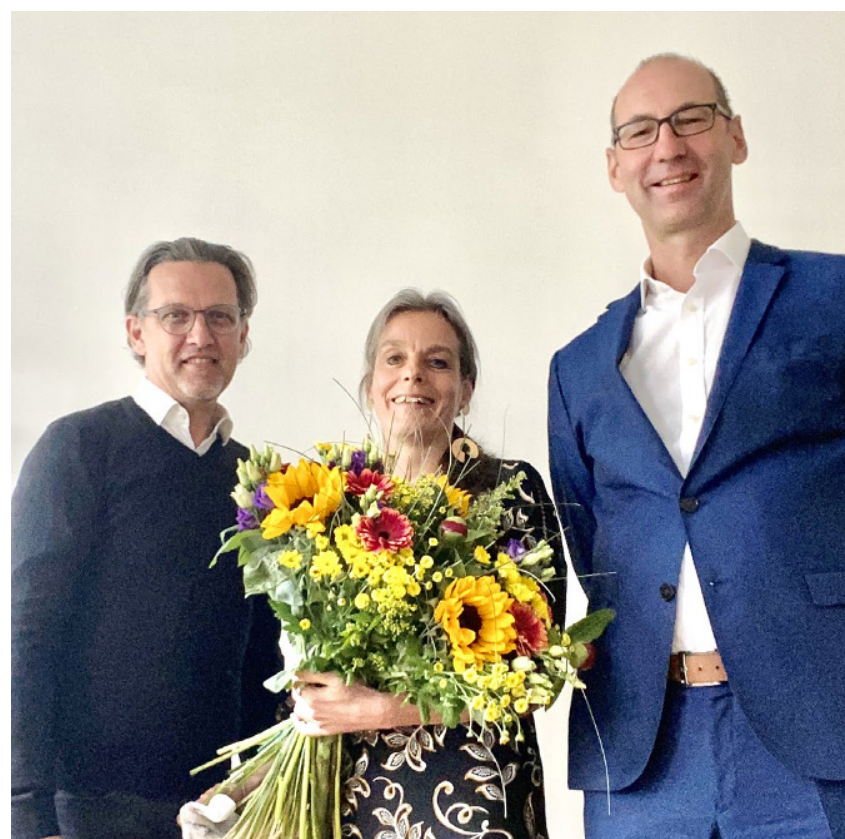

Abb. 1 \ Verabschiedung von Frau Dr. Ing. Monique Jacobs (Mitte) durch Prof. Dr. D. Böckler (links) und Prof. Dr. M. Steinbauer (rechts)
Im Rahmen einer kleinen Zeremonie am 28. April 2020 dankten der Präsident, Prof. Dr. D. Böckler, und Vizepräsident, Prof. Dr. M. Steinbauer, im Namen des Vorstandes und der Mitglieder der DGG, Frau Dr. Jacobs für die geleistete Arbeit und wünschten ihr für die Zukunft alles Gute (- Abb. 1).

Die Geschäfte der Privaten Akademie führte interimistisch Prof. Dr. M. Steinbauer. Die Mitarbeitenden der Geschäftsstelle wurden in dieser Phase von den Mitgliedern des Vorstandes unterstützt, bis am 1.6.2020 Frau Dr. med. Livia Cotta die Geschäftsführung übernahm.

Frau Dr. Cotta (49) war zuletzt als Geschäftsführerin der
Heinrich-Böll-Stiftung für das Management dieser Organisation für politische Bildung verantwortlich. Zuvor war sie als Medizinische Direktorin bei der DKMS - Deutsche Knochenmarkspenderdatei und als Marketingleiterin bei einem Schweizer Medizintechnikunternehmen sowie in verschiedenen Funktionen beim Georg Thieme Verlag tätig. Wir werden Frau Dr. Cotta ausführlich in den nächsten Mitteilungen vorstellen. Der Vorstand freut sich, eine so qualifizierte und sympathische Geschäftsführerin für die DGG und Private Akademie gewonnen zu haben.

\section{Aus dem Vorstand}

Im Rahmen der Vorstandssitzung am 14./15.02.2020 erklärte Heiner Wenk, dass er das Amt des Schatzmeisters aus persönlichen Gründen vorfristig zum Jahresende 2020 abgeben möchte. Der Vorstand äußerte sein Bedauern, aber auch Respekt und Verständnis für diese Entschei- dung und dankte Prof. Wenk sehr für die geleistete Arbeit in dieser wichtigen Funktion. Der Vorstand beschloss, die ohnehin anstehenden Vorstandswahlen im Herbst 2020 um die Wahl eines neuen Schatzmeisters zu ergänzen. 


\section{ABSAGE der 36. Jahrestagung der Deutschen Gesellschaft für Gefäßchirurgie und Gefäßmedizin}

Leider müssen wir die Jahrestagung der DGG im September in Bremen absagen. Nach sorgfältiger Abwägung aller Argumente war es uns nicht möglich, einen Kongress $\mathrm{zu}$ organisieren, der den Anforderungen und berechtigten Interessen der Mitglieder, Teilnehmer, Sponsoren und Organisatoren entsprochen hätte.

Selbst vor dem Hintergrund gelockerter Eindämmungsmaßnahmen und weitestgehend rückläufiger Infektionszahlen der Covid-19-Pandemie konnten sich die zuständigen Landesbehörden in Bremen bis Redaktionsschluss noch nicht verbindlich dazu äußern, ob eine im September stattfindende Tagung mit über 1000 Teilnehmern erlaubt werden kann. Bereits die geltenden Hygienemaßnamen, insbesondere das Abstandsgebot, die Ansteckungsgefahr, Reiseverbote durch Kliniken und Firmen sowie mögliche Quarantäne-Erfordernisse stellen in unseren Augen nicht nur relevante Hindernisse für die Organisati- on dar, sondern würden bereits im Verdachtsfall auf eine Infektion potenziell die gefäßchirurgische Versorgung in Deutschland flächendeckend gefährden. Wir haben daher beschlossen, die diesjährige Tagung abzusagen. Ungeachtet dessen planen wir unsere nächste Jahrestagung im Jahr 2021 vom 13. bis 16. Oktober ebenfalls in Bremen und laden Sie schon jetzt herzlich dazu ein.

Wir hoffen sehr, dass wir mit dieser Entscheidung auf Ihr Verständnis stoßen. Uns ist durchaus bewusst, dass viele von Ihnen bis heute sehr viel Arbeit und Energie in die Kongressvorbereitung investiert und mit Spannung und Vorfreude unsere diesjährige Tagung erwartet haben.

Seien Sie versichert, dass wir bereits an Plänen arbeiten, webbasierte Alternativen ggf. schon dieses Jahr anzubieten. Die Digitalisierung wird künftig auch in unsere Fort- und Weiterbildungsveranstaltungen bleibend Einzug halten.

\section{ACHTUNG, BESONDERE ANKÜNDIGUNG!}

\section{Einladung zur ordentlichen Mitgliederversammlung}

Aufgrund der durch die aktuelle Corona-Pandemie abgesagten Jahrestagung in Bremen, laden wir Sie zur ordentlichen Mitgliederversammlung der DGG am Freitag, den 25. September 2020, 13.30-15.30 Uhr in den Hörsaal 20-5 auf dem Campus des Universitätsklinikums Frankfurt, Theodor-SternKai 7, 60590 Frankfurt ein. nur von außerhalb des Hörsaales erfolgen kann.

Neben den satzungsgemäßen Berichten aus dem Vorstand, stehen dieses Jahr u. a. Wahlen des Vorstandes der DGG e. V. und der Kassenprüfer auf der Tagesordnung. Die Tagesordnungspunkte und Informationen zu den Wahlen der Vorstandsposten sind im Einzelnen aufgeführt. Wir freuen uns, Sie bei unserer ordentlichen Mitgliederversammlung in Frankfurt möglichst zahlreich begrüßen zu können.

\section{Tagesordnung}

1. Begrüßung durch den Präsidenten

2. Feststellung der Tagesordnung
3. Bericht des Präsidenten

4. Bericht aus der Geschäftsstelle

5. Bericht des Sekretärs

6. Bericht des Schatzmeisters

7. Bericht der Kassenprüfer

8. Wahl des Vizepräsidenten (Präsident 2023-2024)

9. Wahl des Schatzmeisters

10. Wahl des Vertreters der niedergelassenen Gefäßchirurgen

11. Wahl des Vertreters der Mitglieder in abhängiger dienstlicher Stellung

12. Wahl des ersten Beisitzers

13. Wahl des zweiten Beisitzers 14. Wahl der Kassenprüfer

15. Verschiedenes

\section{Wahlordnung des Vorstandes der DGG e.V.}

Nur ordentliche Mitglieder sind zur Wahl berechtigt.

Zur Einleitung des Wahlverfahrens unterrichtet der Vorstand zusammen mit der Einladung zur Mitgliederversammlung alle ordentlichen Mitglieder schriftlich über die anstehenden Wahlen und fordert alle ordentlichen Mitglieder auf, innerhalb von drei Wochen nach dieser Ankündigung eigene Kandidaten zu nominieren. Die Unterrichtung der Mitglieder erfolgt durch eine Veröffentlichung in der Vereinszeitschrift Gefässchirurgie und auf der Internet-Seite der DGG. Jedes wahlberechtigte Mitglied kann Wahlvorschläge schriftlich einbringen. Sie müssen von mindestens 10 weiteren wahlberechtigten Mitgliedern aus mindestens zwei unterschiedlichen Institutionen unterschrieben sein. Zusätzlich muss eine schriftliche Bestätigung der vorgeschlagenen Kandidaten vorgelegt werden, in welcher die Bereitschaft zur Kandidatur be- stätigt wird. Die Vorschläge der ordentlichen Mitglieder müssen dem Sekretär der Gesellschaft innerhalb einer Frist von 3 Wochen nach Ankündigung der Wahlen schriftlich per Einschreiben zugegangen sein. Im Anschluss daran werden die Wahlvorschläge des Vorstands und diejenigen der ordentlichen Mitglieder auf der Homepage der DGG mitgeteilt.

Die Wahlen finden auf der Mitgliederversammlung statt. Für jede Wahl bestimmt der Vorstand der Gesellschaft einen Wahlleiter und Wahlhelfer.

Der Wahlleiter stellt fest, welche Anträge zur Wahl gestellt werden und nimmt die Wahlvorschläge entgegen. Er stellt fest, ob der vorgeschlagene Kandidat bereit ist, sich zur Wahl zu stellen; von abwesenden Kandidaten muss diese Erklärung schriftlich vorliegen.

Bei offener Abstimmung ist die Hand hochzuheben. Ergeben sich Zweifel am Abstimmungsergebnis, sind die Stimmen auszu- 
zählen. Bei geheimer Wahl ist der Name des Kandidaten, dem das Mitglied seine Stimme gibt, auf der Wahlkarte einzutragen. Die Auszählung erfolgt durch die Wahlhelfer. Ungültige Stimmkarten bleiben bei der Feststellung des Wahlergebnisses unberücksichtigt. Der Wahlleiter stellt das Ergebnis fest und gibt es der Mitgliederversammlung bekannt.

Gewählt ist der Kandidat mit den meisten Stimmen. Wird im ersten Wahlgang eine absolute Mehrheit nicht erreicht, so ist in einer Stichwahl zwischen den beiden Kandidaten zu entscheiden, die im ersten Wahlgang die meisten Stimmen erhalten haben. Die Stichwahl wird wiederholt, bis ein Kandidat eine absolute Mehrheit erreicht hat.

Der gewählte Kandidat erklärt, ob er die Wahl annimmt.

Bei Gewährleistung der Grundsätze eines ordnungsgemäßen, dokumentierbaren und ggf. geheim durchführbaren Wahlverfahrens, können die Wahlen auch mittels elektronischer Hilfsmittel (bspw. TED) erfolgen.

\section{SommerAkademie 2020}

\begin{abstract}
Nach reiflichen Überlegungen haben Prof. Torsello, Medizinischer Direktor der Privaten Akademie, und der Vorstand beschlossen, die diesjährige Sommerakademie ersatzlos abzusagen. Unter den geltenden Hygienevorschriften wäre es nicht möglich gewesen, diese wichtige Aus-, Fort- und Weiterbildungsveranstaltung unserer Fachgesellschaft zielführend durchzuführen. Mit allen Partnern wurden Lösungen gefunden, die die DGG weder finanziell noch ideell belasten.

Wir danken allen Referenten, Tutoren und Sponsoren, die sich trotz aller Widrigkeiten bereit erklärt hatten, die SommerAkademie aktiv zu unterstützen für das großartige Engagement und allen angemeldeten Teilnehmern für Ihr Verständnis. Alle bereits

entrichteten Teilnahmegebühren werden selbstverständlich zurückerstattet.

Ungeachtet der aktuellen Herausforderungen würden wir uns trotzdem sehr freuen, Sie im nächsten Jahr vom 21.06.25.06.2021 zur nächsten SommerAkademie in Berlin begrüßen zu dürfen.

Die DGG bedankt sich bei Prof. Dr. Giovanni Torsello, der die Sommerakademie vor 5 Jahren ins Leben gerufen hat und mit vielen engagierten Referenten und Kurstutoren zu einer wichtigen gefäßchirurgischen Weiterbildungsveranstaltung etabliert hat, für sein großes Engagement. Die zukünftige wissenschaftliche Leitung wird turnusgemäß Prof. Dr. J. Heckenkamp übernehmen.
\end{abstract}

\section{Nächste Termine - Save The Dates}

\author{
- 15. Konvent der Leitenden \\ Gefäßchirurgen/Gefäßchir- \\ urginnen, Hotel Palace Ber- \\ lin, 22.01.-23.01.2021 \\ - 5. SommerAkademie der \\ DGG e. V., Marriott by Cour- \\ tyard Berlin-Mitte, 21.06.- \\ 25.06.2021 \\ 37. Jahrestagung der DGG \\ e. V., Bremen, 13.10.- \\ 16.10.2021
}

